\title{
Role of system size in freeze-out conditions extracted from transverse momentum spectra of hadrons
}

\author{
Ajay Kumar Dash, ${ }^{1, *}$ Ranbir Singh, ${ }^{1, \dagger}$ Sandeep Chatterjee, ${ }^{2, \ddagger}$ Chitrasen Jena, ${ }^{3, \S}$ and Bedangadas Mohanty ${ }^{1, \|}$ \\ ${ }^{1}$ School of Physical Sciences, National Institute of Science Education and Research, HBNI, Jatni 752050, India \\ ${ }^{2}$ AGH University of Science and Technology, Faculty of Physics and Applied Computer Science, al. Mickiewicza 30, 30-059 Krakow, Poland \\ and Department of Physical Sciences, Indian Institute of Science Education and Research, Berhampur, Transit Campus, Government ITI, \\ Berhampur 760010, Odisha, India \\ ${ }^{3}$ Indian Institute of Science Education and Research, Tirupati 517507, India
}

(Received 13 July 2018; published 5 December 2018)

\begin{abstract}
The data on hadron transverse momentum spectra in different centrality classes of $\mathrm{p}+\mathrm{Pb}$ collisions at $\sqrt{s_{\mathrm{NN}}}=$ $5.02 \mathrm{TeV}$ have been analyzed to extract the freeze-out hypersurface within a simultaneous chemical and kinetic freeze-out scenario. The freeze-out hypersurface has been extracted for three freeze-out schemes that differ in the way strangeness is treated: (i) unified freeze-out for all hadrons at complete thermal equilibrium (1FO), (ii) unified freeze-out for all hadrons with an additional parameter $\gamma_{S}$ which accounts for possible out-of-equilibrium production of strangeness $\left(1 \mathrm{FO}+\gamma_{S}\right)$, and (iii) separate freeze-out for hadrons with and without strangeness content (2FO). Unlike in heavy-ion collisions where $2 \mathrm{FO}$ performs best in describing the mean hadron yields as well as the transverse momentum spectra, with $\mathrm{p}+\mathrm{Pb}$ we find that $1 \mathrm{FO}+\gamma_{S}$ with one fewer parameter than $2 \mathrm{FO}$ performs better. This confirms expectations based on previous analysis of system size dependence in the freeze-out scheme with mean hadron yields: while heavy-ion collisions that are dominated by constituent interactions prefer $2 \mathrm{FO}$, smaller collision systems like proton + nucleus and proton + proton collisions with lesser constituent interaction prefer a unified freeze-out scheme with varying degrees of strangeness equilibration.
\end{abstract}

DOI: 10.1103/PhysRevC.98.064902

\section{INTRODUCTION}

Knowledge of the surface of last scattering of hadrons produced in a heavy-ion collision event is of utmost significance, as it contributes to the calibration of the hadronic physics baseline to be contrasted with data to extract information on the quark gluon plasma phase [1,2] as well as on the QCD critical point $[3,4]$. The hadron resonance gas model has been the main phenomenological model for extraction of the freeze-out hypersurface by comparison to the data on hadron yields [5-10] as well as spectra [11-14]. The surface where the hadrons cease to interact inelastically is known as the chemical freeze-out surface. The hadron yields freeze here. The surface where the hadrons cease to interact even elastically is known as the kinetic freeze-out surface. The shapes of the transverse momentum spectra of hadrons get fixed here. Depending on the model assumptions,

\footnotetext{
*ajayd@niser.ac.in

†ranbir.singh@niser.ac.in

${ }^{\ddagger}$ Sandeep.Chatterjee@ fis.agh.edu.pl

§cjena@iisertirupati.ac.in

"bedanga@niser.ac.in
}

Published by the American Physical Society under the terms of the Creative Commons Attribution 4.0 International license. Further distribution of this work must maintain attribution to the author(s) and the published article's title, journal citation, and DOI. Funded by SCOAP . the chemical and kinetic freeze-out surfaces can be separate [15-17] or together [11,12,18-20]. In this study, we have worked with the THERMINATOR event generator, where a combined freeze-out of both yields and spectra at the same surface is implemented [21,22].

Traditionally, a single unified freeze-out of all hadrons (1FO) has been studied [7-9]. However, data from the Large Hadron Collider (LHC) have thrown open the interpretation of freeze-out and several alternate schemes have been proposed [23-32]. In the standard picture, freeze-out is interpreted as a competition between fireball expansion and interaction of the constituents. Thus it is natural to expect system size dependence under freeze-out conditions, since constituent interactions decrease as one goes from nucleusnucleus $(\mathrm{A}+\mathrm{A})$ to proton-nucleus $(\mathrm{p}+\mathrm{A})$ and proton-proton $(p+p)$ collisions. On the contrary, it was found that 1 FO provides an equally good description of the data on mean hadron yields of $e^{+}+e^{-}, \mathrm{p}+\mathrm{p}$, and $\mathrm{A}+\mathrm{A}$ [33]. This lack of sensitivity of the $1 \mathrm{FO}$ approach to the varying rate of interaction among the constituents and fireball expansion across system size raises doubt about the standard interpretation of freezeout as a competition between expansion and interaction.

In Ref. [34], the yield data were analyzed within three approaches: (i) 1FO, (ii) single unified freeze-out of all hadrons with an additional parameter $\gamma_{S}$ accounting for nonequilibrium production of strangeness $\left(1 \mathrm{FO}+\gamma_{S}\right)$, and (iii) a separate freeze-out surface for hadrons with and without strangeness content (2FO). The data on hadron yield were analyzed across systems- $\mathrm{p}+\mathrm{p}, \mathrm{p}+\mathrm{Pb}$, and $\mathrm{Pb}+\mathrm{Pb}-$ 
enabling one to study the freeze-out condition for midrapidity charged particle multiplicity as well as the system volume varying over three orders of magnitude. It was found that while the $1 \mathrm{FO}$ and $1 \mathrm{FO}+\gamma_{S}$ schemes are blind to system size, 2FO exhibits a strong system size dependence. While for central and midcentral collisions, $2 \mathrm{FO}$ provides the lowest chi-square per degree of freedom, for peripheral $\mathrm{Pb}+\mathrm{Pb}$ to all centralities of $\mathrm{p}+\mathrm{Pb}$ and min bias $\mathrm{p}+\mathrm{p}, 1 \mathrm{FO}+\gamma_{S}$ provides a better description. This emphasizes a plausible freeze-out scenario: in the case of large system sizes, the freeze-out dynamics is dominated by hadron interactions and hence flavor dependence in hadron-hadron cross sections plays a role, resulting in 2FO's being the preferred freeze-out scheme. On the other hand, in small systems the freeze-out is mostly driven by rapid expansion and little interaction, resulting in a sudden and rapid freeze-out and hence disfavoring 2 FO.

In this paper, we extend the above line of argument by studying the data on hadron spectra. The $2 \mathrm{FO}$ prescription has been demonstrated to describe the data on hadron spectra better than $1 \mathrm{FO}$ in $\mathrm{Pb}+\mathrm{Pb}$ at $\sqrt{s_{\mathrm{NN}}}=2.76 \mathrm{TeV}$ [14]. Here we study the data on hadron spectra in $\mathrm{p}+\mathrm{Pb}$ at $\sqrt{s_{\mathrm{NN}}}=$ 5.02 TeV [35-37] and, finally, connect to our previous findings with the spectral data in $\mathrm{Pb}+\mathrm{Pb}$ [14]. The spectra of $\pi^{+}+\pi^{-}, \mathrm{K}^{+}+\mathrm{K}^{-}, \mathrm{p}+\overline{\mathrm{p}}, \phi, \Lambda+\bar{\Lambda}, \Xi+\bar{\Xi}$, and $\Omega+\bar{\Omega}$ are used for this study; they are measured in midrapidity $(0<$ $\left.y_{\mathrm{cm}}<0.5\right)$ by the ALICE Collaboration. We have performed the centrality dependence in this study by analyzing the data in seven centrality classes: $0-5 \%, 5-10 \%, 10-20 \%, 20-40 \%$, 40-60\%, 60-80\%, and 80-100\%

The paper is arranged in the following way. In Sec. II we discuss the model used in this study. The results from the model and data are compared in Sec. III. Finally, we summarize our findings in Sec. IV.

\section{MODEL}

We have studied the data on hadron spectra in three schemes-1FO, $1 \mathrm{FO}+\gamma_{S}$, and $2 \mathrm{FO}$ - using the THERMINATOR event generator $[21,22]$. While $1 F O$ is implemented in the standard version of THERMINATOR, in Ref. [14] the standard version of THERMINATOR was extended to include the $2 \mathrm{FO}$ scheme. We now briefly describe the implementation of the freeze-out hypersurface and the relevant parameters to be extracted in this approach.

The Cooper-Frye prescription provides the hadron spectra emanating from a freeze-out hypersurface,

$$
\frac{d^{2} N}{d y p_{T} d p_{T}}=\int d \Sigma \cdot p f\left(p \cdot u, T, \gamma_{S}, \mu\right),
$$

where $T$ is the temperature, $\mu=\left\{\mu_{B}, \mu_{Q}, \mu_{S}\right\}$ refer to the three chemical potentials corresponding to the three conserved charges of QCD, $u^{\mu}$ is the four-velocity, $d \Sigma^{\mu}$ is the differential element of the freeze-out hypersurface over which the integration in Eq. (1) is supposed to be, and $p$ is the four-momentum. There can be different choices for parametrization of the freeze-out hypersurface and $u^{\mu}$. We have worked within the Krakow model [11], whereby the freeze-out is assumed to occur at a constant proper time $\tau_{f}$,

$$
\tau_{f}^{2}=t^{2}-x^{2}-y^{2}-z^{2}
$$

while $u^{\mu}$ is chosen to be

$$
u^{\mu}=x^{\mu} / \tau_{f},
$$

where $(t, x, y, z)$ is the space-time coordinate.

THERMINATOR accounts for both primary production and the secondary contribution from resonance decays when evaluating the distribution function $f$. The integration in Eq. (1) occurs over the freeze-out hypersurface coordinates, namely, the space-time rapidity $\eta_{s}$, whose integration range is from $-\infty$ to $+\infty$; the azimuthal angle $\phi$, which is integrated from 0 to $2 \pi$; and $\rho=\sqrt{x^{2}+y^{2}}$, the perpendicular distance between the $Z$ axis and the freeze-out hypersurface. $\rho$ is integrated from 0 to $\rho_{\max }$. Thus, we have three parameters within the 1 FO scheme $-T, \tau_{f}$, and $\rho_{\max }$ - to be extracted by comparison with data.

The choice of the thermodynamic ensemble is a relevant topic whenever one discusses system size dependence. In $\mathrm{p}+\mathrm{p}$ collisions at the highest SPS and RHIC energies, the use of a canonical ensemble or a strangeness canonical ensemble has been suggested [38,39]. At the LHC energies, a grand canonical ensemble was found to work best in describing the hadron yields [40]. Similar recent studies on the role of the thermodynamic ensemble in small systems are reported in Refs. [41-43]. Here, we work with the grand canonical ensemble as well. Since we work with the LHC data, which shows a very good particle-antiparticle symmetry, we have set all the chemical potentials to 0 . In $1 \mathrm{FO}+\gamma_{S}$, there is also the additional parameter $\gamma_{S}$ in $f$, which accounts for outof-equilibrium production of strangeness. In $2 \mathrm{FO}$, we have different parameter sets for parametrizing the nonstrange $\left(T_{\mathrm{ns}}\right.$, $\tau_{f_{\mathrm{ns}}}$, and $\left.\rho_{\text {maxns }}\right)$ and strange $\left(T_{\mathrm{s}}, \tau_{f_{\mathrm{s}}}\right.$, and $\left.\rho_{\operatorname{maxs}}\right)$ freeze-out hypersurfaces.

\section{RESULTS}

We have varied $T$ in the range from 145 to $162 \mathrm{MeV}$ in steps of $1-2 \mathrm{MeV}$, whereas $\rho_{\max }$ and $\tau_{f}$ are varied in the range 1.5 to $4.1 \mathrm{fm}$ and 1.5 to $3.1 \mathrm{fm}$, respectively, in steps of $0.1 \mathrm{fm}$. The goodness of the parameter set in describing the data is ascertained from the $\chi^{2} / \mathrm{ndf}$, where

$$
\chi^{2}=\sum_{i}\left(\frac{\operatorname{Data}\left(p_{T_{i}}\right)-\operatorname{Model}\left(p_{T_{i}}\right)}{\operatorname{Error}\left(p_{T_{i}}\right)}\right)^{2},
$$

and ndf $=$ Number of data points - Number of free parameters. Here $\operatorname{Error}\left(p_{T_{i}}\right)$, the denominator, denotes the error in the experimental measurement, where the statistical and systematic errors have been added in quadratures.

The sum goes over all available $\mathrm{p}+\mathrm{Pb}$ data points up to $p_{T}=2.5 \mathrm{GeV} / \mathrm{c}$ [35-37]. For $1 \mathrm{FO}$, we have varied all three parameters, $T, \rho_{\max }$. and $\tau_{f}$, to arrive at the best parameter set. In $1 \mathrm{FO}+\gamma_{S}$, we have also varied $\gamma_{S}$ in the range 0.7 to $1.0 \mathrm{in}$ steps of 0.2 , while in $2 \mathrm{FO}$ we have varied $T, \rho_{\max }$, and $\tau_{f}$ for both nonstrange and strange freeze-out hypersurfaces. The $p_{T}$ spectra as obtained in the model for the different freezeout schemes are compared with data in Fig. 1. In the bottom panel of Figs. 1(a)-1(i), we show the ratio of data to model. Unlike in $\mathrm{Pb}+\mathrm{Pb}$, where there is noticeable disagreement 

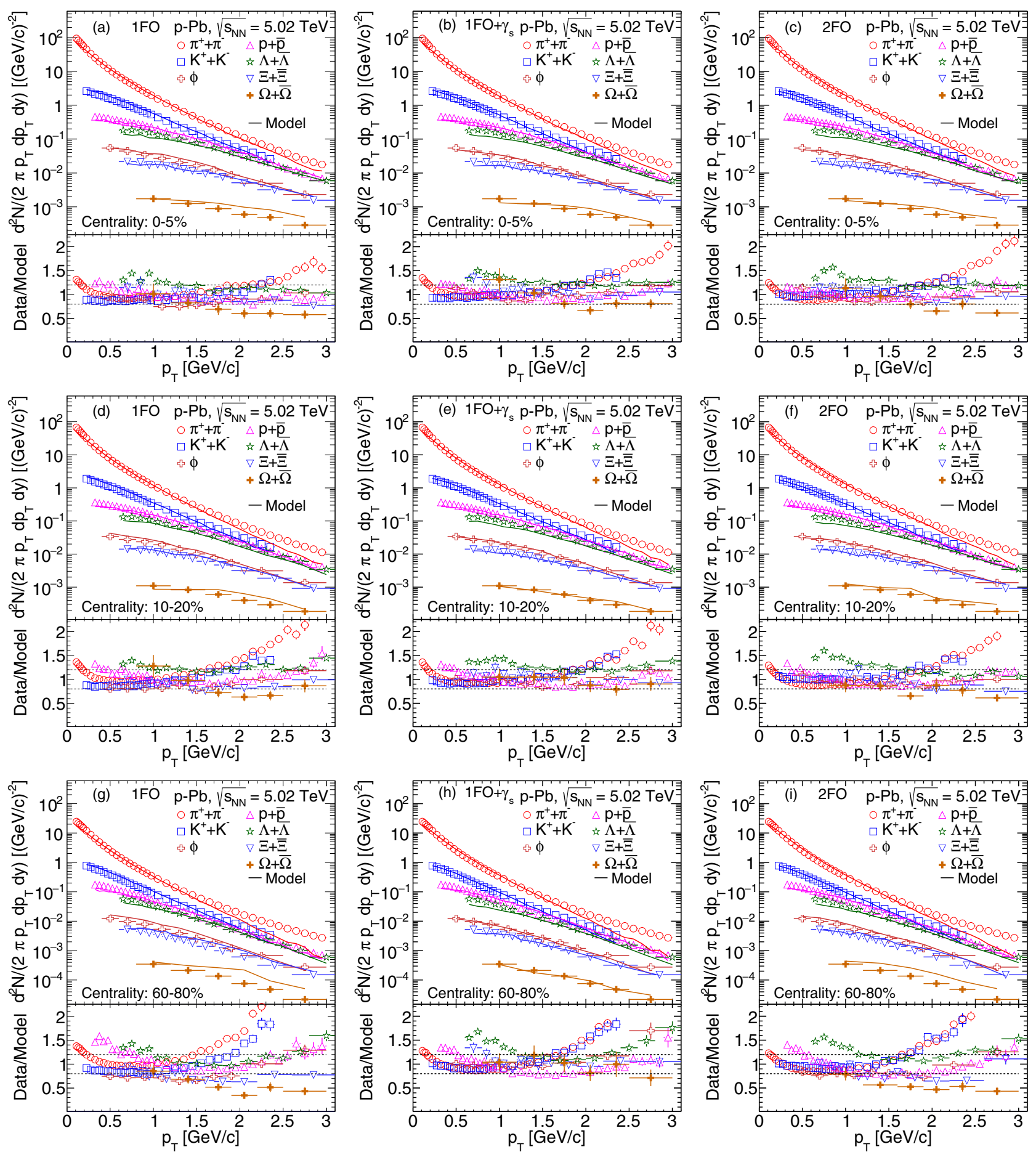

FIG. 1. Comparison of $p_{T}$ spectra in $\mathrm{p}+\mathrm{Pb}$ collisions at $\sqrt{s_{\mathrm{NN}}}=5.02 \mathrm{TeV}$ obtained from the THERMINATOR [21,22] and data [3537] shown for three centralities- $0-5 \%, 10-20 \%$, and 60-80\%-in three FO schemes at $0<y_{\mathrm{cm}}<0.5$. The gross features of the spectral comparison seem to be independent of the freeze-out scheme. The bottom panels (a-i) show the ratio of data to model calculation.

between $1 \mathrm{FO}$ and data, referred to as a proton anomaly, which goes away upon extension of $1 \mathrm{FO}$ to $2 \mathrm{FO}$, in $\mathrm{p}+\mathrm{Pb}$ we do not find any such noteworthy tensions in $1 \mathrm{FO}$. The quality of description of the spectra seems similar overall.

The $\chi^{2} /$ ndf values obtained in the different freeze-out schemes across various centralities are compared in Fig. 2 and the respective values of $\chi^{2}$ and ndf are listed in Table I.
For all centralities, $1 \mathrm{FO}+\gamma_{S}$ provides the lowest $\chi^{2} /$ ndf. The $\chi^{2} /$ ndf grows from around 3 in central collisions to around 10 in the most peripheral bin in this scheme. $\chi^{2} /$ ndf $\sim 1$ is the usual standard for a good statistical description. Thus, overall the $\chi^{2} /$ ndf obtained in our analysis is large. The occurrence of a large $\chi^{2} /$ ndf is quite common in these analyses [14,34,44] and this can be attributed to several factors. The uncertainties 


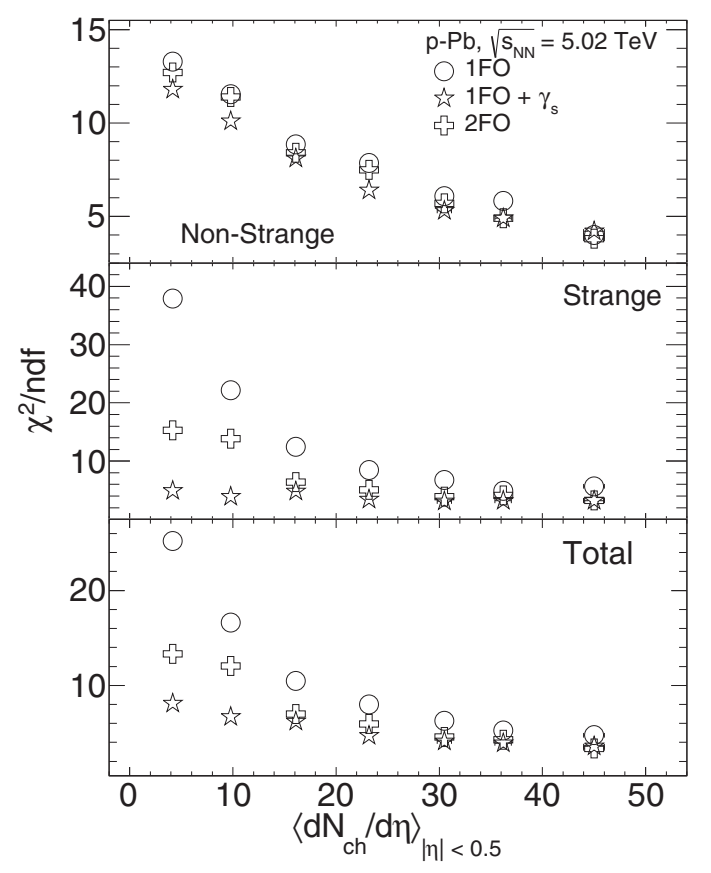

FIG. 2. The $\chi^{2} /$ ndf values are compared for the three freeze-out schemes across central to peripheral collisions in $\mathrm{p}+\mathrm{Pb}$. $1 \mathrm{FO}+\gamma_{S}$ provides the best description across all centralities. The improvement over $1 \mathrm{FO}$ and $2 \mathrm{FO}$ gets better as one goes to more peripheral collisions.

over the hadron spectrum [31,45-48], decay properties of hadrons $[46,48,49]$, and treatment of hadron-hadron interaction $[32,50-53]$ are some of the common sources of systematic improvements of such thermal models. It is usually found that accounting for such effects improves the quality of fits by lowering the $\chi^{2} /$ ndf substantially, keeping the extracted thermal parameters similar $[32,48,49]$. Further, one could also include corrections due to nonthermal physics such as the contribution from the rescattering phase post chemical freeze-out. So the assumption in this work is that even though such corrections will bring the $\chi^{2} /$ ndf down to within the admissible range of unity, the thermal physics of the freezeout hypersurface that we extract here will not change under the incorporation of such systematic improvements of thermal as well as nonthermal effects.
The improvement of $1 \mathrm{FO}+\gamma_{S}$ over $1 \mathrm{FO}$ and $2 \mathrm{FO}$ increases as one goes from central to peripheral collisions. This is driven by the strange sector; it is more sensitive to the three freeze-out schemes studied here, which differ in the treatment of the freeze-out of strange hadrons. The yield in the nonstrange sector receives a partial contribution from the decays of strange resonances. This leads to a small sensitivity in the fit quality of the nonstrange sector to the different freeze-out schemes studied here. The improvement in the nonstrange sector with $1 \mathrm{FO}+\gamma_{S}$ is mild and uniform across centralities. We have enlisted the best parameter values that describe the transverse momentum spectra across different centralities within the three freeze-out schemes in Table II.

Finally, in Fig. 3 we have plotted the extracted freeze-out parameters corresponding to the lowest $\chi^{2} /$ ndf with event multiplicity across different centralities in $\mathrm{p}+\mathrm{Pb}$ and $\mathrm{Pb}+$ $\mathrm{Pb}$ that vary over three orders of magnitude. While $T$ remains mostly flat between 145 and $160 \mathrm{MeV}, \rho_{\max }$ and $\tau_{f}$ show an increase of 5-7 times. The growth rate is smooth across system size. We also note that the difference between nonstrange and strange freeze-out parameters systematically increase as we go to events with a higher multiplicity, signifying the role of interaction. However, currently the uncertainties over the extracted parameters in the nonstrange and strange sectors are large and do not allow us to further quantify the magnitude of the hierarchy in freeze-out of the strange and nonstrange flavors. $\gamma_{S}$ in $\mathrm{p}+\mathrm{Pb}$ steadily increases from 0.74 to about 0.94 across peripheral to central collisions. The approach to strangeness equilibration with more central $\mathrm{p}+\mathrm{Pb}$ events could be related to the larger entropy deposition in the initial state in central $\mathrm{p}+\mathrm{Pb}$ collisions as opposed to peripheral events [54]. We use similar errors in $T, \rho_{\max }$, and $\tau_{f}$ as for $\mathrm{Pb}+\mathrm{Pb}$ results [14] since the errors are mostly system size independent.

\section{SUMMARY AND OUTLOOK}

The hadron yields and $p_{T}$ spectra are the standard observables to shed light on freeze-out dynamics. Contrary to expectations, the 1FO scheme is known to be blind to system size dependence in freeze-out [33]. However, simultaneous analysis of the hadron yields in $\mathrm{Pb}+\mathrm{Pb}, \mathrm{p}+\mathrm{Pb}$, and $p+p$ revealed an interesting system size dependence of the

TABLE I. $\chi^{2}$ and ndf in the $1 \mathrm{FO}, 1 \mathrm{FO}+\gamma_{s}$, and $2 \mathrm{FO}$ schemes in $\mathrm{p}+\mathrm{Pb}$ collisions at $\sqrt{s_{\mathrm{NN}}}=5.02 \mathrm{TeV}$.

\begin{tabular}{|c|c|c|c|c|c|c|c|c|c|}
\hline Centrality (\%) & \multicolumn{9}{|c|}{$\chi^{2}(\mathrm{ndf})$} \\
\hline $0-5$ & $248(62)$ & $374(66)$ & $622(131)$ & $256(61)$ & $208(65)$ & $464(130)$ & $225(59)$ & $206(63)$ & $432(128)$ \\
\hline $5-10$ & $361(62)$ & $325(66)$ & $686(131)$ & $297(61)$ & $207(65)$ & $504(130)$ & $290(59)$ & $257(63)$ & $547(128)$ \\
\hline $10-20$ & $377(62)$ & $445(66)$ & $822(131)$ & $324(61)$ & $204(65)$ & $528(130)$ & $338(59)$ & $246(63)$ & $584(128)$ \\
\hline $40-60$ & $549(62)$ & $822(66)$ & 1371(131) & $494(61)$ & $315(65)$ & $808(130)$ & $495(59)$ & $401(63)$ & $896(128)$ \\
\hline $60-80$ & $716(62)$ & $1463(66)$ & $2179(131)$ & $615(61)$ & $251(65)$ & $866(130)$ & 671(59) & $873(63)$ & $1544(128)$ \\
\hline $80-100$ & $824(62)$ & $2428(64)$ & $3253(129)$ & $721(61)$ & $311(63)$ & $1032(128)$ & $746(59)$ & $931(61)$ & $1677(126)$ \\
\hline
\end{tabular}


TABLE II. Thermal freeze-out parameters in the $1 \mathrm{FO}, 1 \mathrm{FO}+\gamma_{s}$, and $2 \mathrm{FO}$ schemes in $\mathrm{p}+\mathrm{Pb}$ collisions at $\sqrt{s_{\mathrm{NN}}}=5.02 \mathrm{TeV}$. The average error in $T$ and $\gamma_{s}$ is $2 \mathrm{MeV}$ and 0.02 , respectively, whereas the error in $\rho_{\max }$ and $\tau_{f}$ is around $15 \%$ for all centrality classes.

\begin{tabular}{|c|c|c|c|c|c|c|c|c|c|c|}
\hline Centrality (\%) & \multicolumn{4}{|c|}{$1 \mathrm{FO}\left(1 \mathrm{FO}+\gamma_{s}\right)$} & \multicolumn{3}{|c|}{ Strange } & \multicolumn{3}{|c|}{ Nonstrange } \\
\hline $0-5$ & $157(158)$ & $3.9(3.8)$ & $2.7(2.7)$ & 0.94 & 160 & 3.6 & 2.5 & 154 & 4.1 & 3.0 \\
\hline $5-10$ & $157(158)$ & $3.5(3.5)$ & $2.6(2.6)$ & 0.92 & 160 & 3.3 & 2.3 & 154 & 3.8 & 2.8 \\
\hline $10-20$ & $157(157)$ & $3.3(3.4)$ & $2.5(2.5)$ & 0.90 & 160 & 3.1 & 2.2 & 154 & 3.6 & 2.7 \\
\hline $40-60$ & $155(156)$ & $2.7(2.7)$ & $2.2(2.3)$ & 0.84 & 157 & 2.5 & 2.0 & 153 & 2.85 & 2.35 \\
\hline $60-80$ & $155(155)$ & $2.2(2.3)$ & $2.0(2.1)$ & 0.80 & 156 & 2.1 & 1.9 & 153 & 2.4 & 2.2 \\
\hline $80-100$ & $154(153)$ & $1.6(1.7)$ & $1.9(1.9)$ & 0.74 & 155 & 1.5 & 1.7 & 153 & 1.7 & 1.9 \\
\hline
\end{tabular}

preferred freeze-out scheme: $2 \mathrm{FO}$ is preferred over $1 \mathrm{FO}$ and $1 \mathrm{FO}+\gamma_{S}$ in $\mathrm{Pb}+\mathrm{Pb}$, while in small systems like $\mathrm{p}+\mathrm{Pb}$ and $\mathrm{p}+\mathrm{p}, 1 \mathrm{FO}+\gamma_{S}$ is preferred [34]. In order to put this hypothesis on a stronger footing, here we extend the previous analysis to hadron spectra. While $2 \mathrm{FO}$ is known to describe the hadron spectra better in $\mathrm{Pb}+\mathrm{Pb}$, here we analyze the data for different centralities in $\mathrm{p}+\mathrm{Pb}$. We find that allowing for a different hypersurface for the freeze-out of the strange hadrons does not improve the quality of the fits. This is in accordance with our previous study of hadron yields [34]. Thus, our current analysis with the data on hadron spectra reaffirms the hypothesis on the system size dependence of the freeze-out scheme: a flavor-dependent freeze-out scheme is preferred in large systems, while unified freeze-out is preferred in small
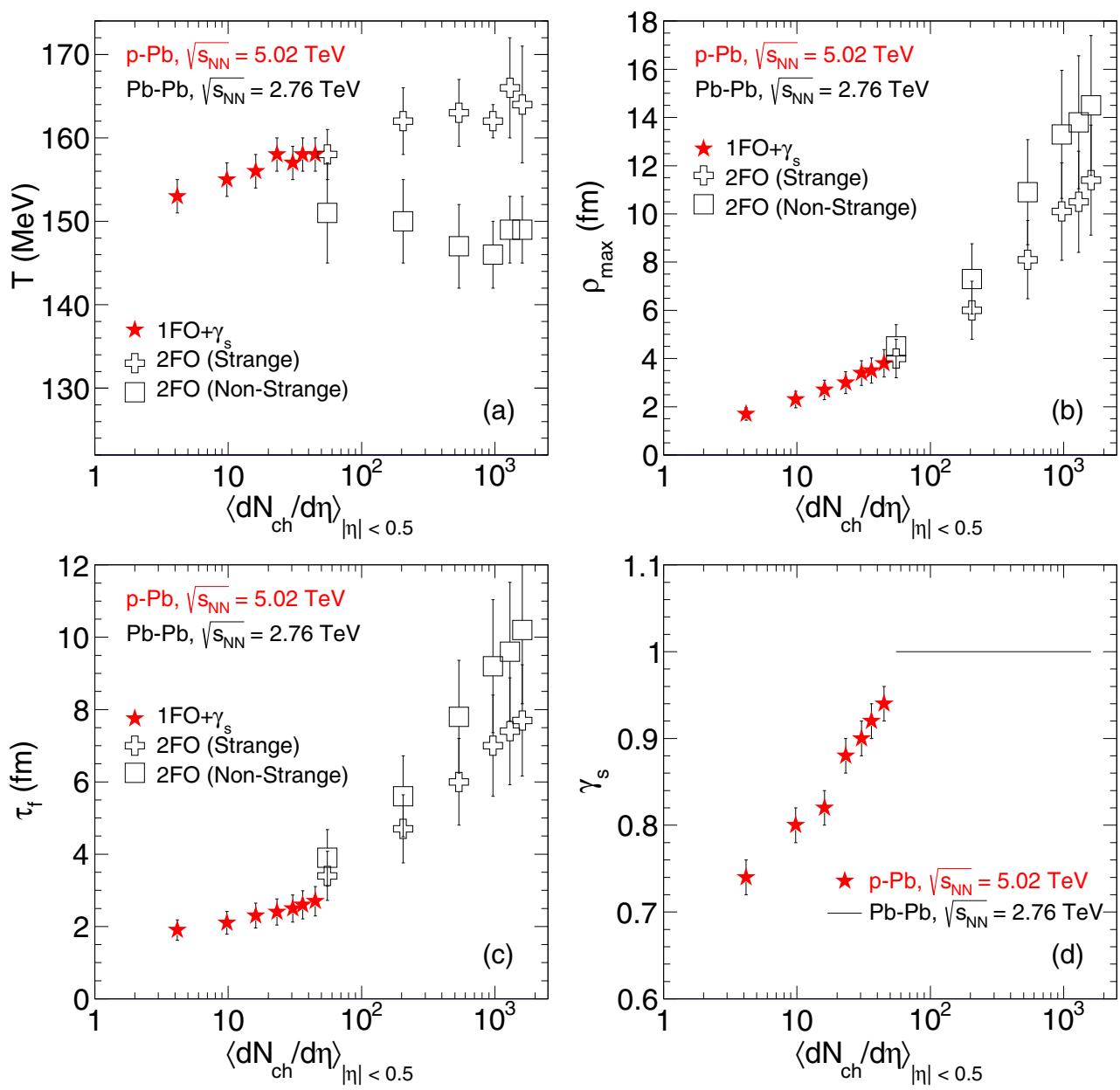

FIG. 3. The extracted freeze-out parameters with the best goodness of fit for different centralities in $\mathrm{p}+\mathrm{Pb}$ and $\mathrm{Pb}+\mathrm{Pb}$ collisions. There is a gradual preference for sequential freeze-out of the strange and nonstrange flavors as we go to higher multiplicity events. The $\gamma_{S}$ value is fixed at 1 for $\mathrm{Pb}+\mathrm{Pb}$ collisions at $\sqrt{s}_{\mathrm{NN}}=2.76 \mathrm{TeV}$. 
systems. Thus, the role of interaction in larger systems is mostly to delay the freeze-out of nonstrange hadrons.

\section{ACKNOWLEDGMENTS}

A.K.D. and R.S. acknowledge the support of XIIth Plan Project No. 12-R\&D-NIS-5.11-0300 of the Government of
India. B.M. acknowledges financial support from the J. C. Bose National Fellowship of DST, Government of India. S.C. acknowledges the support of AGH UST statutory task No. 11.11.220.01/1 within the subsidy of the Ministry of Science and Higher Education and by National Science Centre Grant No. 2015/17/B/ST2/00101.
[1] M. Gyulassy and L. McLerran, Nucl. Phys. A750, 30 (2005).

[2] J. Adams et al. (STAR Collaboration), Nucl. Phys. A757, 102 (2005).

[3] K. Rajagopal and F. Wilczek, in At the Frontier of Particle Physics, edited by M. Shifman (World Scientific, Singapore, 2000), Vol. 3, pp. 2061-2151.

[4] M. A. Stephanov, Prog. Theor. Phys. Suppl. 153, 139 (2004); International Journal of Modern Physics A (World Scientific, Singapore, 2005), Vol. 20, pp. 4387-4392.

[5] P. Braun-Munzinger, J. Stachel, J. P. Wessels, and N. Xu, Phys. Lett. B365, 1 (1996).

[6] G. D. Yen and M. I. Gorenstein, Phys. Rev. C 59, 2788 (1999).

[7] J. Cleymans and K. Redlich, Phys. Rev. C 60, 054908 (1999).

[8] F. Becattini, J. Cleymans, A. Keranen, E. Suhonen, and K. Redlich, Phys. Rev. C 64, 024901 (2001).

[9] A. Andronic, P. Braun-Munzinger, and J. Stachel, Nucl. Phys. A 772, 167 (2006).

[10] S. Chatterjee, S. Das, L. Kumar, D. Mishra, B. Mohanty, R. Sahoo, and N. Sharma, Adv. High Energy Phys. 2015, 349013 (2015).

[11] W. Broniowski and W. Florkowski, Phys. Rev. Lett. 87, 272302 (2001).

[12] W. Broniowski and W. Florkowski, Phys. Rev. C 65, 064905 (2002).

[13] V. Begun, W. Florkowski, and M. Rybczynski, Phys. Rev. C 90, 014906 (2014).

[14] S. Chatterjee, B. Mohanty, and R. Singh, Phys. Rev. C 92, 024917 (2015).

[15] E. Schnedermann, J. Sollfrank, and U. W. Heinz, Phys. Rev. C 48, 2462 (1993).

[16] M. van Leeuwen et al., Nucl. Phys. A 715, 161 (2003).

[17] J. M. Burward-Hoy (PHENIX Collaboration), Nucl. Phys. A 715, 498 (2003).

[18] J. Letessier, J. Rafelski, and A. Tounsi, Phys. Lett. B 328, 499 (1994).

[19] T. Csorgo and L. P. Csernai, Phys. Lett. B 333, 494 (1994).

[20] L. P. Csernai and I. N. Mishustin, Phys. Rev. Lett. 74, 5005 (1995).

[21] A. Kisiel, T. Taluc, W. Broniowski, and W. Florkowski, Comput. Phys. Commun. 174, 669 (2006).

[22] M. Chojnacki, A. Kisiel, W. Florkowski, and W. Broniowski, Comput. Phys. Commun. 183, 746 (2012).

[23] J. Steinheimer, J. Aichelin, and M. Bleicher, Phys. Rev. Lett. 110, 042501 (2013).

[24] F. Becattini, M. Bleicher, T. Kollegger, T. Schuster, J. Steinheimer, and R. Stock, Phys. Rev. Lett. 111, 082302 (2013).

[25] M. Petrán, J. Letessier, V. Petráček, and J. Rafelski, Phys. Rev. C 88, 034907 (2013).

[26] R. Bellwied, S. Borsanyi, Z. Fodor, S. D. Katz, and C. Ratti, Phys. Rev. Lett. 111, 202302 (2013).
[27] S. Chatterjee, R. M. Godbole, and S. Gupta, Phys. Lett. B 727, 554 (2013).

[28] K. A. Bugaev, D. R. Oliinychenko, J. Cleymans, A. I. Ivanytskyi, I. N. Mishustin, E. G. Nikonov, and V. V. Sagun, Europhys. Lett. 104, 22002 (2013).

[29] M. Floris, Nucl. Phys. A 931, 103 (2014).

[30] J. Noronha-Hostler and C. Greiner, Nucl. Phys. A931, 1108 (2014).

[31] A. Bazavov, H. T. Ding, P. Hegde, O. Kaczmarek, F. Karsch, E. Laermann, Y. Maezawa, S. Mukherjee, H. Ohno, P. Petreczky, C. Schmidt, S. Sharma, W. Soeldner, and M. Wagner, Phys. Rev. Lett. 113, 072001 (2014).

[32] P. Alba, V. Vovchenko, M. I. Gorenstein, and H. Stoecker, Nucl. Phys. A 974, 22 (2018).

[33] F. Becattini, P. Castorina, A. Milov, and H. Satz, Eur. Phys. J. C 66, 377 (2010).

[34] S. Chatterjee, A. K. Dash, and B. Mohanty, J. Phys. G 44, 105106 (2017).

[35] B. B. Abelev et al. (ALICE Collaboration), Phys. Lett. B 728, 25 (2014).

[36] J. Adam et al. (ALICE Collaboration), Phys. Lett. B 758, 389 (2016)

[37] J. Adam et al. (ALICE Collaboration), Eur. Phys. J. C 76, 245 (2016).

[38] I. Kraus, J. Cleymans, H. Oeschler, K. Redlich, and S. Wheaton, Phys. Rev. C 76, 064903 (2007).

[39] I. Kraus, J. Cleymans, H. Oeschler, and K. Redlich, Phys. Rev. C 79, 014901 (2009).

[40] S. Das, D. Mishra, S. Chatterjee, and B. Mohanty, Phys. Rev. C 95, 014912 (2017).

[41] V. V. Begun, V. Vovchenko, M. I. Gorenstein, and H. Stoecker, arXiv:1805.01901.

[42] N. Sharma, J. Cleymans, and L. Kumar, Eur. Phys. J. C 78, 288 (2018).

[43] N. Sharma, J. Cleymans, and B. Hippolyte, arXiv:1803.05409.

[44] V. Begun, W. Florkowski, and M. Rybczynski, Phys. Rev. C 90, 054912 (2014)

[45] W. Broniowski and W. Florkowski, Phys. Lett. B 490, 223 (2000)

[46] S. Chatterjee, R. M. Godbole, and S. Gupta, Phys. Rev. C 81, 044907 (2010).

[47] P. Alba, R. Bellwied, S. Borsányi, Z. Fodor, J. Günther, S. D. Katz, V. Mantovani Sarti, J. Noronha-Hostler, P. Parotto, A. Pasztor, I. P. Vazquez, and C. Ratti, Phys. Rev. D 96, 034517 (2017).

[48] S. Chatterjee, D. Mishra, B. Mohanty, and S. Samanta, Phys. Rev. C 96, 054907 (2017).

[49] V. Vovchenko, M. I. Gorenstein, and H. Stoecker, Phys. Rev. C 98, 034906 (2018)

[50] V. Vovchenko, A. Pasztor, Z. Fodor, S. D. Katz, and H. Stoecker, Phys. Lett. B 775, 71 (2017). 
[51] V. Vovchenko, M. I. Gorenstein, and H. Stoecker, Phys. Rev. Lett. 118, 182301 (2017).

[52] P. Huovinen and P. Petreczky, Phys. Lett. B 777, 125 (2018).
[53] A. Dash, S. Samanta, and B. Mohanty, Phys. Rev. C 97, 055208 (2018).

[54] P. Castorina, S. Plumari, and H. Satz, Intl. J. Mod. Phys. E 26, 1750081 (2017). 